\title{
Study of the Pressure Drop and Flow Field in Standard Gas Cyclone Models Using the Granular Model
}

\author{
Nabil Kharoua, ${ }^{1}$ Lyes Khezzar, ${ }^{1}$ and Zoubir Nemouchi ${ }^{2}$ \\ ${ }^{1}$ Department of Mechanical Engineering, Petroleum Institute, Abu Dhabi, P.O. Box 2533, United Arab Emirates \\ ${ }^{2}$ Département de Génie Mécanique, Faculté des Sciences de l'Ingénieur, Université Mentouri Constantine, Constantine 25017, Algeria
}

Correspondence should be addressed to Nabil Kharoua, nkharoua@pi.ac.ae

Received 17 March 2011; Accepted 3 May 2011

Academic Editor: Fernando T. Pinho

Copyright (C 2011 Nabil Kharoua et al. This is an open access article distributed under the Creative Commons Attribution License, which permits unrestricted use, distribution, and reproduction in any medium, provided the original work is properly cited.

\begin{abstract}
A particle-laden flow inside solid gas cyclones has been studied using computational fluid dynamics (CFD). The effects of high temperatures and different particle loadings have been investigated. The Reynolds stress (RSM) model-predicted results, in the case of pure gas, are within engineering accuracy even at high temperatures. Using the granular mixture model for the cases of particle-laden flow, discrepancies occurred at relatively high loadings (up to $0.5 \mathrm{~kg} / \mathrm{m}^{3}$ ). Since the pressure drop is strongly related to the friction inside the cyclone body, the concept of entropy generation has been employed to detect regions of high frictional effects. Friction has been observed to be important at the vortex finder wall, the bottom of the conical-part wall, and the interface separating the outer and the core streams. The discrepancies between the present numerical simulation and the experimental results taken from the existing literature, which are caused by the mixture and turbulence models simplifying assumptions, are discussed in this paper.
\end{abstract}

\section{Introduction}

Solid gas cyclone separators are known to have low cost, they are easy to operate, simple to manufacture, and relatively easy to maintain because of lack of moving parts. This has made them the best devices used in industries where the separation of solid particles from a gaseous phase is crucial. Cyclone separators are used in several industrial applications such as cement industry, pressurized fluidized bed combustors, and fluidized catalytic cracking processes.

The highly swirling flow generated inside the cyclone causes the particles to be ejected under the effect of the centrifugal force towards the outer wall. On the other hand, the generation of high swirl intensities requires high energy consumption (high pressure drop) [1-3]. The design of cyclones involves thus two conflicting and simultaneous requirements of minimizing the pressure drop and maximizing the separation efficiency. The pressure drop and separation efficiency are thus important for successful design and need to be predicted with the required engineering accuracy.

Theoretical studies have established semiempirical models for the prediction of single-phase pressure drop [4-6].
The main parameters used are the geometry of the cyclone and a characteristic tangential velocity component. The single-phase reasoning has been developed to take into account the effects of the solid loading and the temperature. The influence of temperature is introduced via the density and viscosity in the Reynolds number while the loading effect is usually taken care of with the aid of correction factors of the single-phase case using the inlet dust loading as a parameter. The semiempirical relations in the presence of two-phase flow are not always able to predict the pressure drop correctly. Cortés and Gil [5] in their review list a series of pressure drop correlations, and recently Chen and Shi [7] established a "universal" model to calculate cyclone pressure drop including the effect of solid loading, and it appears to offer superior performance in comparison with previously proposed correlations.

Theoretical models, by their nature, are restricted to the particular practical cases for which they were tested. Computational fluid dynamics (CFD) which can represent a viable tool for engineering analysis of cyclone flows does not have such a limitation. Until now, simulation of cyclone flows is still a challenging task due to the presence of swirl increasing 
the anisotropy of the turbulence and requiring at least a full second moment closure type for the Reynolds averaged equations to deal with the complexity of such flows. In addition, particle-laden flows still being a challenge introduce another level of difficulty. Several studies have addressed CFD modeling [8-16] since the pioneering work of Boysan et al. [8]. Ivanov et al. [17] used the algebraic slip model in Fluent where they considered a monodisperse particle population and ignored particle-particle interaction. Wang et al. [14] used the full Reynolds stress model in conjunction with the stochastic Lagrangian multiphase model in Fluent on a coarse mesh of around 48,000 cells. Although CFD models for both turbulence and multiphase flows are being constantly improved for robustness and accuracy, their success in predicting cyclone flows remains mitigated [18]. Qian et al. [19] simulated high $\left(0.5-2 \mathrm{~kg} / \mathrm{m}^{3}\right.$ of carrying fluid) inlet concentration of particles using the ASMM (algebraic slip mixture model) contrary to the general trend of using the Lagrangian-Eulerian model in most of the CFD studies. Different particle sizes were considered by creating additional secondary phases and an optimum collision coefficient of 0.98 was established from their experiments. The authors mentioned convergence problems when trying to represent the size distribution by more than four secondary phases. Shalaby et al. [16] have combined the large eddy simulation turbulence model (LES) with the Lagrangian approach for the multiphase flow in a one-way coupling mode. Derksen et al. [20] conducted a more elaborate LES study including the two-way coupling interaction reflecting the effects of the particles on the primary gaseous phase. They made some assumptions which are the considerations at the cyclone bottom for the escaping particles, the omission of particleparticle collisions, the limitation of the effect of particles on the resolved field of the gaseous phase, the uniformly sized particles in addition to the forcing of the overflow boundary condition, by placing a small disk just upstream of the upper outlet, to avoid the backward effect on the solution in the internal domain. They confirmed the effects of the mass loading on the cyclone efficiency through its effects on the mean and turbulent flow fields. The particles have a damping effect on the mean flow field of the carrying gaseous phase which is expected to reduce the separation efficiency while the attenuation of the turbulent field results in a decreased particle dispersion promoting, thus, their ejection towards the cyclone walls where they are separated. It, therefore, appears that the accuracy of pressure drop prediction using CFD in cyclones under two-phase conditions remain still debatable, in view of the complexity of the flow.

This paper presents a systematic study on the numerical prediction of pressure drop of a series of cyclone models of different geometries and inlet conditions obtained from the literature [7] using the granular mixture model to predict the pressure drop under various temperatures and several solid loading conditions. The full Reynolds stress model (RSM) was used to close the Reynolds averaged equations of motion. The models used in this work represent a compromise between accuracy and computational effort. In particular, the model performance under high solid loading conditions and the setting of boundary conditions (wall functions) and convergence criteria are discussed in this paper.

\section{Mathematical and Numerical Model}

In the single-phase computations, the steady form of the Reynolds averaged Navier-Stokes equations for three-dimensional flow is solved using the finite-volume technique implemented in the commercial code Fluent.

The mixture model $[21,22]$ is used to model the flow of the air and monodispersed solid particles mixture. The two phases are allowed to move at different velocities, using the concept of slip velocity. The model solves a single system of continuity and momentum equations for the mixture of the two phases. The secondary solid phase is characterized by its relative velocity and its volume fraction computed from additional equations. The model assumes that the secondary phase is uniformly dispersed and reaches its terminal velocity in a short time and that the phases are in local equilibrium over a small length scale. Therefore, it is suitable for flows in which a strong coupling between the phases exists such as in cyclones. It is noteworthy to mention that no general agreement on the appropriate submodels included in the granular mixture model exist in the literature. In the present study, Syamlal and O'Brien [23] model has been used for the granular viscosity and the drag coefficient calculation. An algebraic equation obtained by neglecting the convective and the diffusive terms in the transport equation derived from the kinetic theory [24], served for the calculation of granular temperature. The solids pressure and the radial distribution have been predicted via the Lun et al. [25] model. The packing limit of 0.63 recommended for monodispersed particles was used. A value of 0.98 for the coefficient of restitution was used [19].

The continuity equation for the mixture is

$$
\nabla \cdot(\rho \vec{v})=0
$$

where $\vec{v}$ is the mass-averaged velocity

$$
\vec{v}=\frac{\left(x_{a} \rho_{a} \vec{v}_{a}+x_{s} \rho_{s} \vec{v}_{s}\right)}{\rho}=y_{a} \vec{v}_{a}+y_{s} \vec{v}_{s}
$$

$\rho$ is the mixture density

$$
\rho=x_{a} \rho_{a}+x_{s} \rho_{s}
$$

$\rho_{a}$ and $\rho_{s}$ are the densities of the air (primary phase) and the particles (secondary phase), respectively, $x_{a}$ and $x_{s}$ the volume fractions, $y_{a}$ and $y_{s}$ the mass fractions, and $\vec{v}_{a}$ and $\vec{v}_{s}$ the velocities.

The momentum equation for the mixture is

$$
\begin{gathered}
\nabla(\rho \vec{v} \vec{v})=-\nabla p+\nabla \cdot\left[\left(\mu+\mu_{t}\right)\left(\nabla \vec{v}+\nabla \vec{v}^{T}\right)\right] \\
+\rho \vec{g}-\nabla \cdot\left(x_{s} \rho_{s} \vec{v}_{\mathrm{dr}, s} \vec{v}_{\mathrm{dr}, s}\right),
\end{gathered}
$$

$\mu$ and $\mu_{t}$ are the laminar and turbulent viscosities of the mixture. 
$\vec{v}_{\mathrm{dr}, \mathrm{s}}$ is the drift velocity of the particles (relative to the mass-averaged velocity of the mixture) given by

$$
\vec{v}_{\mathrm{dr}, s}=\vec{v}_{s}-\vec{v}
$$

The solid particles are allowed to move at a different velocity from that of the gaseous phase, using the concept of slip velocity (relative velocity of the solid particles phase to that of the gaseous phase). An algebraic slip formulation is used in conjunction with the mixture model.

The slip velocity is

$$
\vec{v}_{s, s}=\vec{v}_{s}-\vec{v}_{a}
$$

The drift velocity can be expressed in terms of the slip velocity as follows:

$$
\vec{v}_{\mathrm{dr}, s}=y_{a} \vec{v}_{s, s} .
$$

The algebraic form of the slip velocity [21], based on the assumption that local equilibrium between the phases is reached over a short distance, can be expressed as follows:

$$
\vec{v}_{s, s}=\tau_{s}\left[\frac{\left(\rho-\rho_{s}\right)}{\left(\rho_{s} f_{\text {drag }}\right)}\right] \vec{a}-\left(\frac{\mu_{t}}{\rho x_{s} \sigma_{D}}\right) \nabla x_{s},
$$

$f_{\text {drag }}$ is the drag coefficient (see [18]).

$\vec{a}$ is the secondary phase acceleration

$$
\vec{a}=\vec{g}-(\vec{v} \cdot \nabla) \vec{v} .
$$

The second term on the right-hand side represents the diffusion due to turbulent fluctuations. $\sigma_{D}$ is the Prandtl dispersion coefficient, and $\tau_{s}$ is the particle relaxation time which can be written as

$$
\tau_{s}=\frac{\left(\rho_{s} d^{2}\right)}{\left(18 \mu_{a}\right)},
$$

where $d$ is the particle diameter and $\mu_{a}$ the primary phase viscosity [22].

In the modified form of the mixture model for solids, the granular viscosity of the suspension is function of the particle loading and contains three contributions among which the kinetic part represents the viscosity at very low loadings ( $<10^{-6} \%$ by volume) allowing the particles to move without any collisions, the collisional part represents the viscosity at medium concentration $\left(>10^{-6} \%\right.$ and $<10^{-3} \%$ by volume) where the distance between the particles is reducing and collisions become possible, and the frictional part which represents the viscosity at high concentrations causing friction between the particles rather than collisions. The last contribution was omitted in the present work due to the moderate concentrations used of less than 0.0004 by volume corresponding to a dilute flow [26];

$$
\begin{gathered}
\mu_{s}=\mu_{s, \mathrm{col}}+\mu_{s, \mathrm{kin}}, \\
\mu_{s, \mathrm{col}}=\frac{4}{5} x_{s} \rho_{s} d_{s} g_{0, s s}\left(1+e_{s s}\right)\left(\frac{\theta_{s}}{\pi}\right)^{1 / 2}, \\
\mu_{s, \mathrm{kin}}=\left[\frac{\left(x_{s} \rho_{s} d_{s}\left(\theta_{s} \pi\right)^{1 / 2}\right)}{6\left(3-e_{s s}\right)}\right]\left[1+\frac{2}{5}\left(1+e_{s s}\right)\left(3 e_{s s}-1\right)\right] x_{s} g_{0, s s},
\end{gathered}
$$

where $g_{0, s s}$ is the nondimensional distance between the particles called the radial distribution and is given by

$$
g_{0, s s}=\left[1-\left(\frac{x_{s}}{x_{s, \max }}\right)^{1 / 3}\right]^{-1},
$$

and $e_{s s}$ is the coefficient of restitution reflecting the collisional behavior of the particles.

The granular temperature $\theta_{s}$ has an algebraic equation

$$
\left(-p_{s} \overline{\bar{I}}+\overline{\overline{\tau_{s}}}\right): \nabla \vec{v}_{s}-\gamma \theta_{s}+\Phi_{a s}=0,
$$

where $\left(-p_{s} \overline{\bar{I}}+\overline{\overline{\tau_{s}}}\right): \nabla \vec{v}_{s}$ is the generation of energy due to the solids stress tensor, $\gamma \theta_{s}$ is the collisional dissipation of energy, and $\Phi_{a s}$ the energy exchange between the gas/solid or solid/solid phases.

The collisional dissipation of energy represents the rate of energy dissipation due to collisions between particles. Lun et al. [25] proposed the following model:

$$
\gamma \theta_{s}=\left[\frac{\left(12\left(1-e_{s s}^{2}\right) g_{0, s s}\right)}{\left(d_{s} \sqrt{\pi}\right)}\right] \rho_{s} x_{s} \theta_{s}^{3 / 2}
$$

$\Phi_{a s}$ is the energy exchange [27]

$$
\Phi_{a s}=-3 K_{a s} \theta_{s} .
$$

The exchange coefficient $K_{a s}$ can be written in the following form:

$$
K_{a s}=\frac{x_{s} \rho_{s} f_{\mathrm{drag}}}{\tau_{s}},
$$

where $f_{\text {drag }}$ is the drag function and $\tau_{s}$ the particulate relaxation time defined as

$$
\tau_{s}=\frac{\left(\rho_{s} d_{s}^{2}\right)}{\left(18 \mu_{a}\right)}
$$

where $d_{s}$ is the diameter of the particles.

According to [23]

$$
f_{\text {drag }}=\frac{\left(C_{D} \operatorname{Re}_{s} x_{a}\right)}{\left(24 v_{r, s}^{2}\right)},
$$

where the drag coefficient [28] is

$$
C_{D}=\left(0.63+\frac{4.8}{\left(\operatorname{Re}_{s} / v_{r, s}\right)}\right)^{2} .
$$

The terminal velocities of particles $v_{r, s}$ are a function of the volume fraction $x_{a}$ and relative Reynolds number $\operatorname{Re}_{s}$ [29]

$$
\operatorname{Re}_{s}=\frac{\left(\rho_{s} d_{s}\left|\vec{v}_{s}-\vec{v}_{a}\right|\right)}{\mu_{a}} .
$$

The solid pressure should also be taken into account in the mixture momentum equations

$$
p_{s}=x_{s} \rho_{s} \theta_{s}+2\left(1+e_{s s}\right) g_{0, s s} x_{s}^{2} \rho_{s} \theta_{s} .
$$




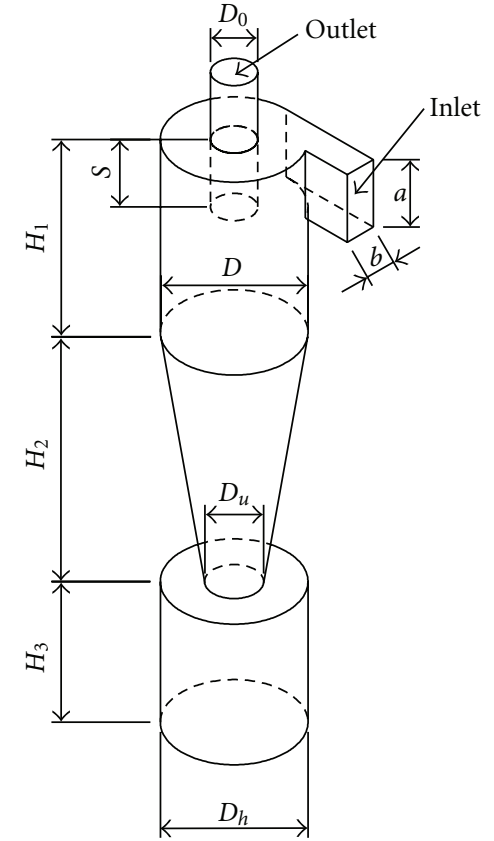

Figure 1: Geometry of conventional cyclone.

The secondary-phase mass fraction is calculated from its transport equation

$$
\left(\rho y_{s} \vec{v}\right)=-\nabla\left[\rho y_{s}\left(1-y_{s}\right) \vec{v}_{s, s}\right]
$$

where the diffusion of the particles by turbulent fluctuations is taken into account in the slip velocity expression.

The Reynolds stress model [30] is used to capture the turbulence effects in the flow. The computations were performed using the steady segregated solver. The simple algorithm, the third-order Quick scheme, and PRESTO were used for the velocity-pressure coupling, convective terms discretization, and pressure interpolation, respectively.

In addition to the residuals, the static pressure was monitored at appropriate locations to ensure convergence when a stabilized solution was observed. Residuals were in the range $10^{-3}$ to $10^{-4}$. An average of 15,000 iterations was necessary to obtain convergence.

The mixture used was composed of solid particles of Talcum powder 325 mesh $\left(\rho=2750 \mathrm{~kg} / \mathrm{m}^{3}, 8\right.$ microns mean diameter), carried by air. The dimensionless pressure drop form (Euler number), is defined by

$$
\xi=\frac{\Delta p}{\left(0.5 \rho v_{\text {in }}^{2}\right)} .
$$

\section{Geometry and Boundary Conditions}

The geometries of the cyclones used [7] consist of the four conventional configurations of Stairmand, Stern, Lapple, and PV with different sizes as shown in Figure 1 and Table 1.

The number of computational cells constituting the mesh ranged from 30,500 to 230,000 cells to provide a compromise between accuracy and computational cost, and these are

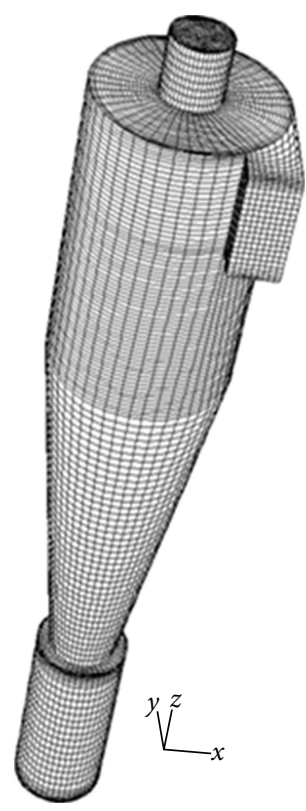

Figure 2: Computational mesh.

given in Table 2. Figure 2 shows an example of the hexahedral mesh used in this work. The grid sensitivity test is important to ensure the independence of the final solution of the number of computational cells. The case with $0.01 \mathrm{~kg} / \mathrm{m}^{3}$ solids loading is taken as a sample of the grid sensitivity test conducted for the present study. Figure 3 illustrates the tangential velocity profiles obtained with two different meshes (coarse $=112376$ cells, fine $=230224$ cells $)$ at three axial positions in the conical part and in the swirl chamber. The reference of the axial direction $z$ is the cyclone bottom. The profiles present slight deviations whose effects on the cyclone performance should be negligible.

At the inlet, a prescribed velocity, turbulence intensity and hydraulic diameter $\left(D_{h}\right)$ for turbulence, and volume fraction and granular temperature for particles have been imposed. The approach used to the turbulence parameters at the inlet is based on the theory of fully developed duct flows. For the cases considered, the turbulence intensity based on the Reynolds number at the inlet of the cyclone ranged from 3 to 3.3. Then, the turbulent kinetic energy and its dissipation rate are derived from the turbulence intensity and the turbulent length scale known from the dimensions of the cyclone inlet. The remaining Reynolds stresses are obtained from the turbulent kinetic energy assuming an isotopic turbulence at the inlet of the cyclone with equal normal stresses and zero shear stresses. On the walls the standard wall function was used [31].

\section{Results and Discussion}

The results are organized in three sections. In the first section the computations of several pure gas single-phase cases for seven different cyclone geometries and varying inlet conditions are presented. The second part contains results treating the single gaseous-phase under the effect of 
TABLE 1: Cyclone normalized dimensions.

\begin{tabular}{lcccccccccc}
\hline Geometry & Diam $(\mathrm{m})$ & $a / D$ & $b / D$ & $D_{0} / D$ & $D_{u} / D$ & $D_{h} / D$ & $S / D$ & $H_{1} / D$ & $H_{2} / D$ & $H_{3} / D$ \\
\hline Stairmand & 0.305 & 0.50 & 0.20 & 0.50 & 0.375 & 0.544 & 0.5 & 1.5 & 2.5 & 1.18 \\
Stern & 0.335 & 0.61 & 0.32 & 0.56 & 0.400 & 0.544 & 0.91 & 1.4 & 1.3 & 1.18 \\
Lapple1 & 0.287 & 0.53 & 0.23 & 0.52 & 0.250 & 0.544 & 1.60 & 2.1 & 2.2 \\
Lapple2 & 0.287 & 0.53 & 0.11 & 0.52 & 0.250 & 0.544 & 1.60 & 2.1 & 2.2 & 1.18 \\
PV1 & 0.300 & 0.56 & 0.25 & 0.32 & 0.400 & 0.544 & 0.56 & 1.6 & 2.2 & 1.18 \\
PV2 & 0.400 & 0.56 & 0.26 & 0.32 & 0.400 & 0.544 & 0.56 & 1.6 & 2.2 \\
PV3 & 0.600 & 0.56 & 0.26 & 0.32 & 0.400 & 0.544 & 0.56 & 1.6 & 2.2 & 1.18 \\
PV4 & 0.600 & 0.49 & 0.22 & 0.32 & 0.400 & 0.544 & 0.49 & 1.6 & 2.2 & 1.18 \\
\hline
\end{tabular}

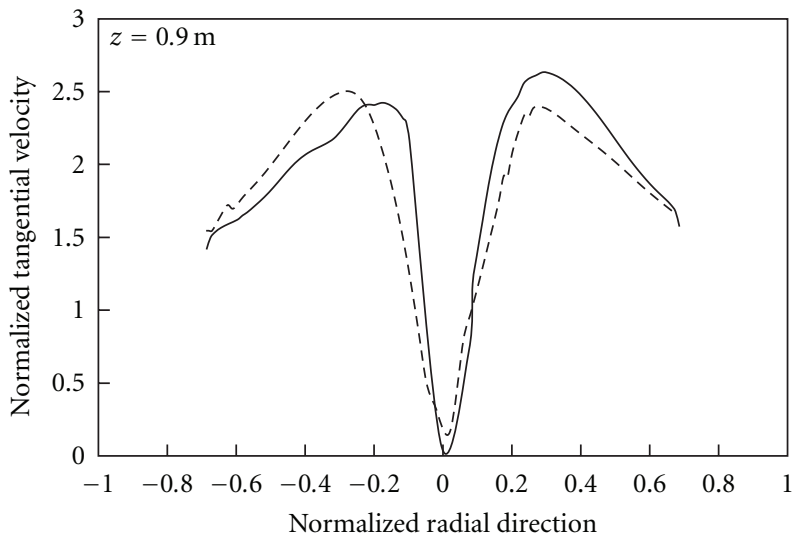

(a)

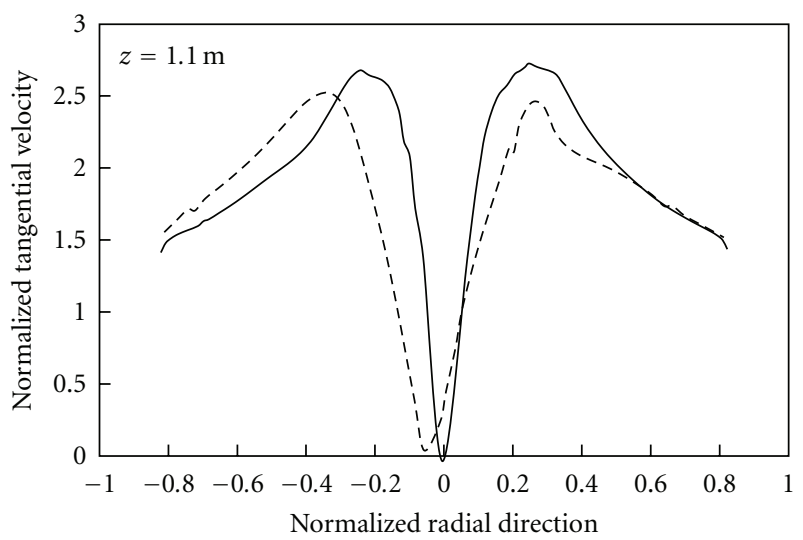

(b)

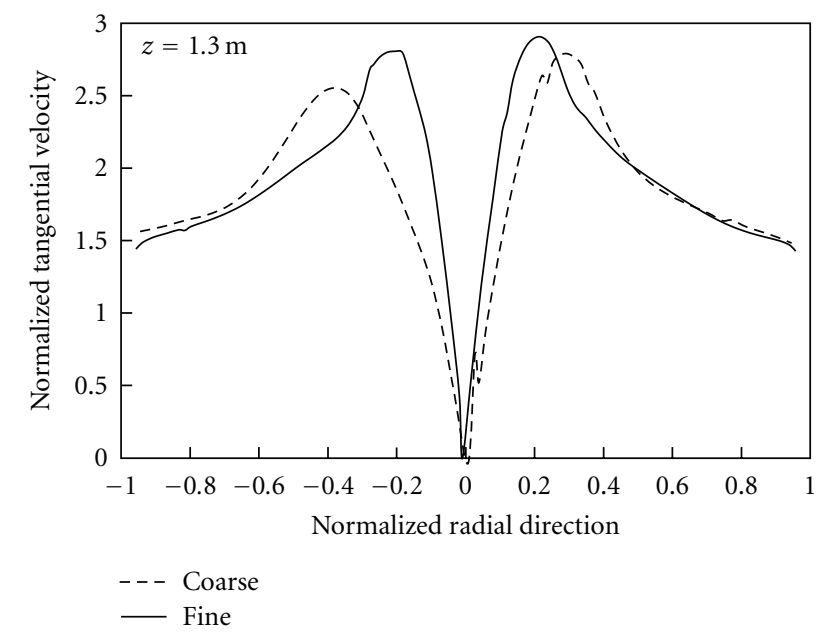

(c)

FIGURE 3: Radial profiles of the tangential velocity component at different positions in the axial direction $z$ of the geometry PV2 (coarse mesh $=112376$ cells, fine mesh $=230224$ cells $)$.

different temperatures and inlet velocities for a single cyclone geometry. The third section includes the effect of solid loading on the pressure drop for a single geometry. For all the cases studied, the CFD results are compared with experimental results and empirical models available in the literature.

4.1. Pressure Drop with a Pure Gas Phase. Table 2 recapitulates the pressure drop results in nondimensional form ob- tained using CFD and compared to the experimental results [7] and those obtained using Chen's theoretical-model for the same cases.

As can be seen, the pressure drop for the pure-gas case is successfully predicted. The difference between numerical and experimental results was in the range $0.6-15 \%$. In the absence of particles, the pressure drop is a function of geometrical parameters and the swirl intensity. Chen and Shi [7] showed that the swirl intensity can be represented by the 
TABLE 2: Pressure drop coefficient for pure gas.

\begin{tabular}{lccccc}
\hline Geometry & $V_{i}(\mathrm{~m} / \mathrm{s})$ & Grid & $\xi_{\text {th }}$ & $\xi_{\exp }$ & $\xi_{\text {CFD }}$ \\
\hline Stairmand & 5.05 & 30544 & 5.90 & 5.68 & 5.63 \\
Stern & 16.07 & 35020 & 8.45 & 7.25 & 8.36 \\
Lapple1 & 14.97 & 52682 & 5.49 & 7.19 & 6.783 \\
Lapple2 & 28.42 & 33836 & 3.65 & 3.68 & 3.748 \\
PV1 & 20.0 & 64632 & 20.85 & 20.3 & 20.86 \\
PV2 & 18.89 & 230224 & 22.22 & 21.74 & 21.88 \\
PV3 & 14.98 & 120963 & 22.85 & 22.56 & 20.75 \\
\hline
\end{tabular}

tangential velocity-component at the wall of the cylindrical section. Figure 4 demonstrates how well CFD predicts the tangential velocity profiles compared to the experimental results [32] at different axial positions (along $\mathrm{z}$ axis) starting from the cyclone bottom. The two first profiles are in the swirl chamber at $z=594 \mathrm{~mm}$ and $z=654 \mathrm{~mm}$, respectively, while the third profile is in middle of the conical part at $z=204 \mathrm{~mm}$.

In conventional analysis the pressure drop is usually attributed to three contributions, the expansion and the contraction loss at the inlet region and at the bottom of the vortex finder, respectively, the fluid-wall friction, and the dissipation of the dynamic head in the vortex finder (Cortés and Gil [5]; Chen and Shi [7]).

In this paper, the expression of entropy generation due to friction [33] has been used to detect regions of high friction inside the cyclone. The energy dissipated by friction can be obtained by simply multiplying equation (24) by the constant temperature. In general, the entropy generation is expressed in terms of fluid friction irreversibility which is the term presented in this study and the heat transfer irreversibility which contribution is nil for such isothermal flows. The entropy generation $\dot{S}$ due to friction in whole of the flow domain was obtained using the equation below with the velocity being that of the mixture,

$$
\dot{S}=\left(\frac{\left(\mu+\mu_{t}\right)}{T}\right)\left[\left(\frac{\partial v_{i}}{\partial x_{j}}\right)+\left(\frac{\partial v_{j}}{\partial \mathrm{x}_{i}}\right)\right]\left(\frac{\partial v_{i}}{\partial x_{j}}\right) .
$$

It can be seen, from Figure 5, that the entropy generation is more important at the vortex finder walls and at its entry which is a well known region of high-velocity gradients and turbulence generation in cyclones.

The interface separating the downwardly directed outer stream and the upwardly directed inner stream (interface of zero axial velocity) appeared to be an additional important source of entropy generation.

4.2. Effect of Temperature on Pressure Drop. As the temperature increases, the air density decreases and its viscosity increases giving lower Reynolds numbers and hence lower maximums of the tangential-velocity components for the same inlet velocity compared to the case of ambient temperature. Table 3 contains results of the pressure drop obtained starting from an ambient temperature and reaching $973 \mathrm{~K}$. The results show generally good agreement although, in some cases, errors reached up to $18 \%$. The case of $973 \mathrm{~K}$
TABle 3: Pressure drop coefficient for different temperatures (Case PV1 of Table 1).

\begin{tabular}{lcccccc}
\hline$T(\mathrm{~K}) \rho\left(\mathrm{kg} / \mathrm{m}^{3}\right)$ & $\mu(\mathrm{kg} / \mathrm{ms}) \times 10^{-5}$ & $v_{i}(\mathrm{~m} / \mathrm{s})$ & $\xi_{\text {th }}$ & $\xi_{\exp }$ & $\xi_{\text {CFD }}$ \\
\hline 470 & 0.69 & 2.6115 & 19.62 & 18.52 & 19.3 & 18.43 \\
685 & 0.49 & 3.368 & 20.11 & 17.0 & 18.4 & 15.08 \\
676 & 0.49 & 3.368 & 16.14 & 16.50 & 18.4 & 16.64 \\
973 & 0.34 & 4.1994 & 36.29 & 16.98 & 17.4 & 15.06 \\
\hline
\end{tabular}

and $36.29 \mathrm{~m} / \mathrm{s}$ shows the opposing effects of increased temperature and inlet velocity. The high inlet velocity is expected to cause higher pressure drop, but the effect of very high temperature could not be overcome.

4.3. Effects of Solid Phase on Pressure Drop. Contrary to the two previous cases computed, problems of solution instability have been encountered especially at relatively high solids loadings $\left(0.5 \mathrm{~kg} / \mathrm{m}^{3}\right.$ of fluid). Results obtained using the RSM and the Granular mixture models are presented, and the difficulties and probable causes of discrepancies are discussed.

It has been stated in the literature that in such flows, the particles ejected outwards contribute in increasing the frictional effect of the wall [5]. This, normally, causes an increase of the pressure drop but the swirl decay under the same effect of friction causes the pressure drop to decrease rather than increase.

The total entropy generation inside the cyclone for the case of dust-laden flow (concentration $0.05 \mathrm{~kg} / \mathrm{m}^{3}$ ) has reduced to half compared with pure gas for the same geometry and inlet conditions from 0.00671 to $0.00388 \mathrm{~W} / \mathrm{K}$. Indeed, the flow damping causes the attenuation of the velocity gradients, hence the frictional effects, as can be observed in Figure 6.

The distribution of the particles volume fraction feed concentrations of $0.01 \mathrm{~kg} / \mathrm{m}^{3}$ and $1 \mathrm{~kg} / \mathrm{m}^{3}$ (Figures 7,8 , 9 , and 10) in the cyclone-wall region and in a plane crossing the cyclone body at $y=0$ is examined. It can be seen that high concentrations of particles are situated near the cyclone wall corresponding to the separated particles especially in the corners adjacent to the recirculation zones where the velocities are low. For low loading $\left(0.01 \mathrm{~kg} / \mathrm{m}^{3}\right)$, even with the agglomeration of the particles near the wall, the concentration remained very low with a maximum of 0.000238 . In the case of relatively high loading $\left(1 \mathrm{~kg} / \mathrm{m}^{3}\right)$, the concentration near the wall reached nearly $25 \%$ at the corner corresponding to the junction between the cylinder and the cone. Higher particle concentrations are also observed in the conical part. Qian et al. [19] have found similar results for particles with diameters of 5 and 10 microns. Near zero particles volume fraction exists in the hopper in Figures 9 and 10 because it has been voluntarily emptied as explained below in this section.

Figure 11 illustrates the variation of the pressure drop with the inlet solid loading. Qian et al. [19] have outlined, albeit in a crude way, the complete trend of the pressure drop where a critical load for which the decreasing trend inverts and mentioned the difficulty they encountered to 


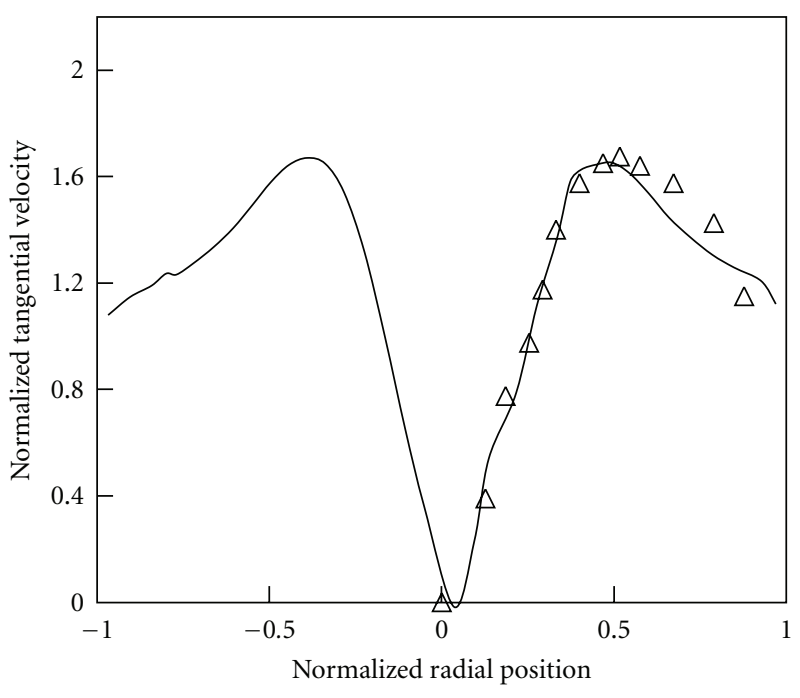

(a)

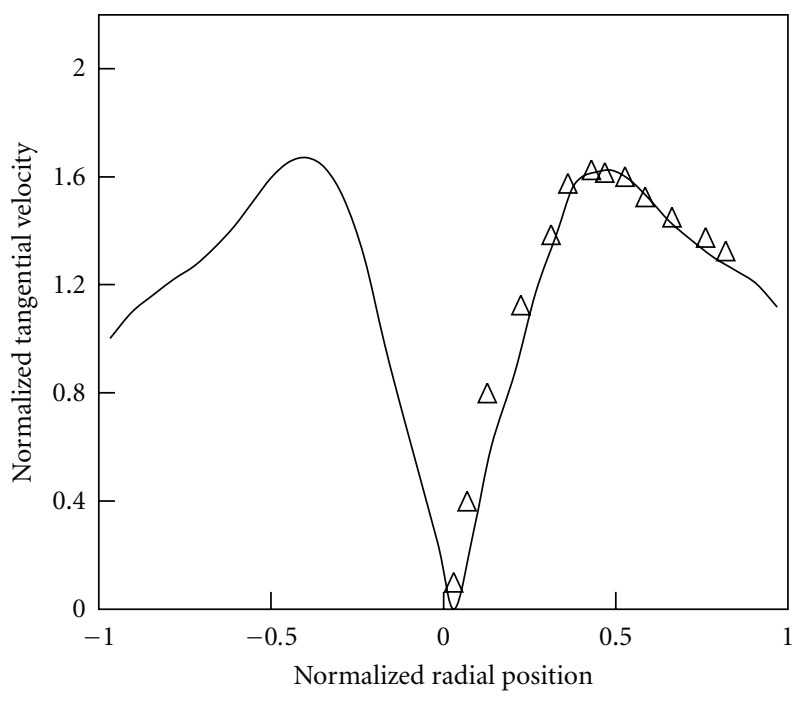

(b)

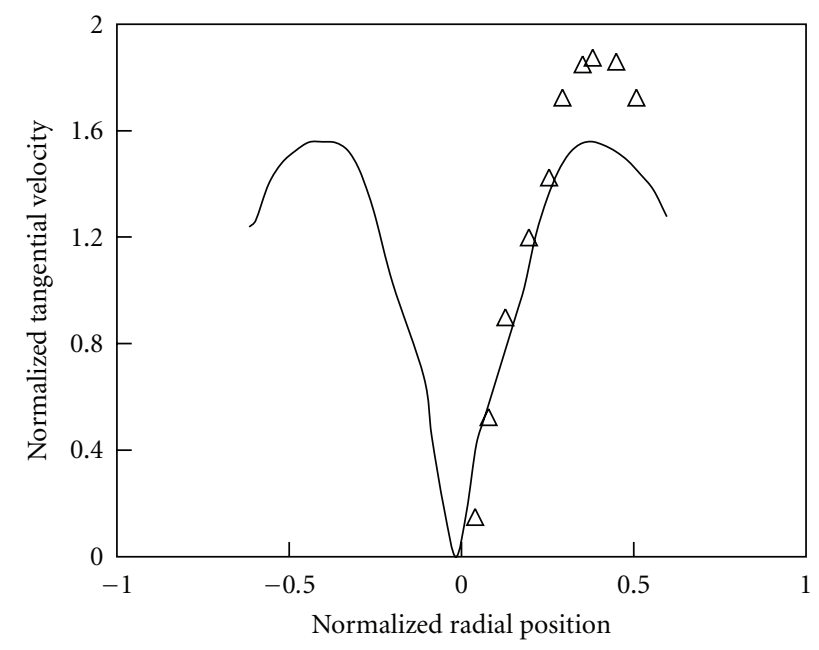

$\triangle$ Experimental - RSM

(c)

FIGURE 4: Radial profiles of the tangential velocity (a) and (b) in the swirl chamber, (c) in the conical part (experimental results from [32]).

predict it correctly because of the limitation of the granular model they used at high loadings. The predicted results, using two models [7, 34], showed that except for the highest loadings greater than $0.5 \mathrm{~kg} / \mathrm{m}^{3}$, CFD calculations are close to the Baskakov model and do capture the trend for the other loadings. The model of [34] detected a critical loading nearly equal to $0.2 \mathrm{~kg} / \mathrm{m}^{3}$ at which the pressure drop inverts its trend. The results corresponding to a concentration of 0.5 and $1 \mathrm{~kg} / \mathrm{m}^{3}$ obtained using CFD show an important discrepancy with the experimental and the empirical models results. This can be attributed to the limitations of the mixture model at relatively high concentrations which are still a challenging task for CFD models in general $[6,18]$. In fact, for moderate to dense loadings (more then $10^{-3}$ by volume), the particles act on the continuous phase by damping the mean and turbulent flows. Furthermore, particles collisions become more probable which in turn affects their speed and direction of motion, thus affecting their momentum. While the pressure drop could be successfully predicted for only pure gas even at high temperatures [13, 16, 32], many previous studies have failed to predict the pressure drop correctly when the effect of the particles on the primary phase became important $[19,32]$.

The comparison of radial profiles of the tangential velocity component (Figure 6) at the same axial position located in the cylindrical section, obtained with different particle loadings, illustrates how the inlet concentration of solids affects the swirl motion. Unfortunately, experimental measurements of the tangential velocity could not be found to estimate the discrepancy engendered at high particle loadings.

The multiphase model simplifying assumptions may contribute strongly in explaining the discrepancies between the CFD and the experimental results. The assumption of 

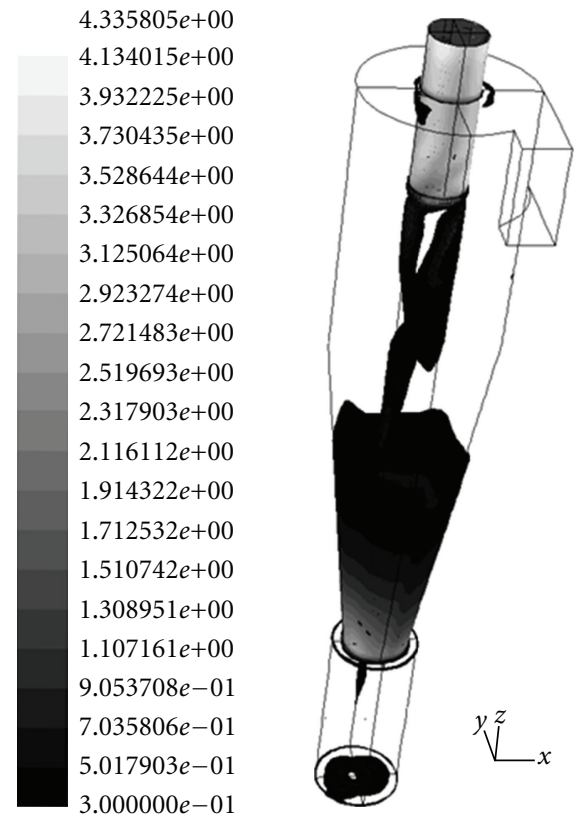

FIGURE 5: Contours of entropy generation for a pure-gas case (case PV2 in Table 2).

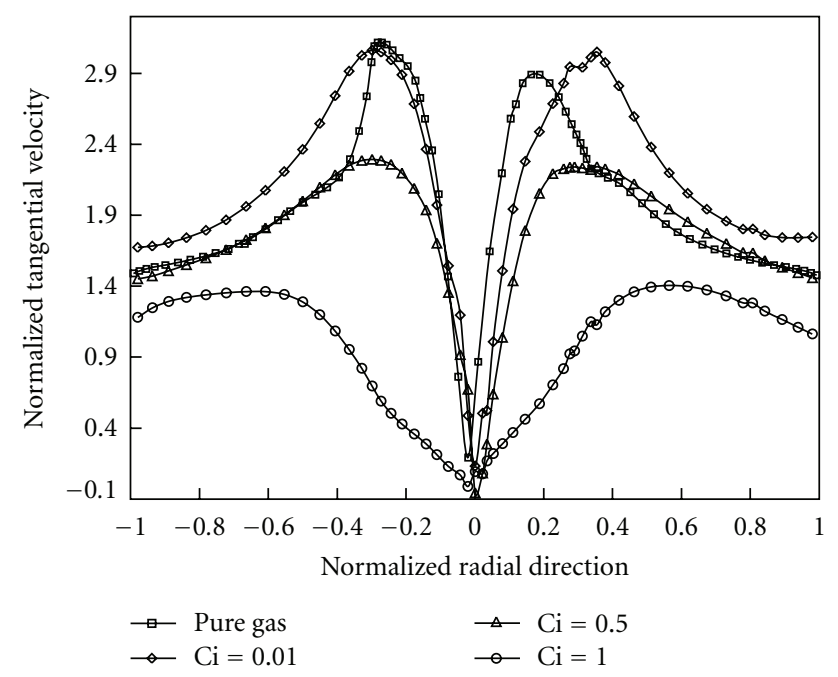

FIGURE 6: Effect of the particle loading on the tangential velocity.

monodispersed particles leads to a different particles behavior and hence different effect on the gaseous phase. Qian et al. [19] attempted to take into account the size distribution in their simulation work, using the granular mixture model, by creating several solid phases with different average size. They could not obtain a converged solution with more than four secondary phases.

In almost all of the previous studies, the effect of the particles on the gaseous phase (two-way coupling) and the particle-particle interactions were not considered which is, strictly speaking, acceptable only for dilute flows. The forces usually taken into account are the centrifugal, the drag, and

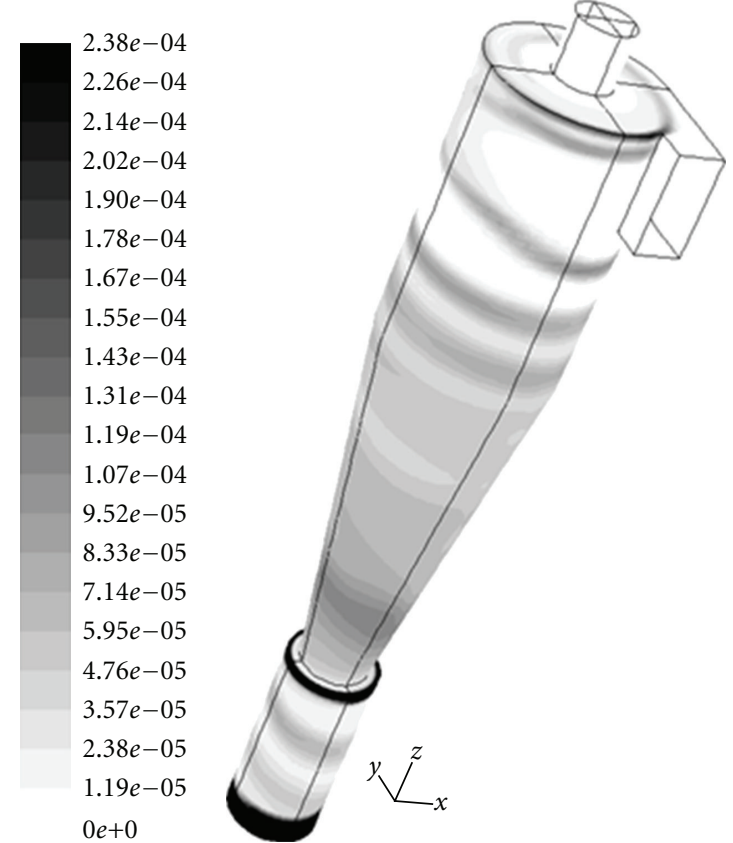

FIgURE 7: Particles volume fraction near the cyclone wall $\left(0.01 \mathrm{~kg} / \mathrm{m}^{3}\right)$.
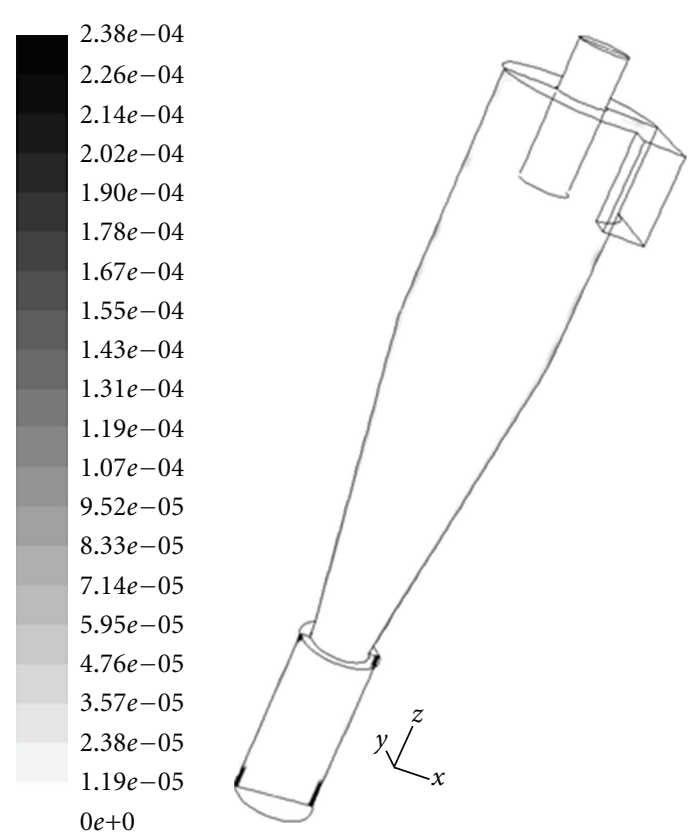

Figure 8: Particles volume fraction at $y=0\left(0.01 \mathrm{~kg} / \mathrm{m}^{3}\right)$.

the gravitational forces. Xiaodong et al. [35] found that the Saffman lift force could accelerate the separation of small particles and shorten their residence time. In addition, the assumption of spherical shape can alter the force balance applied on the particles. In other words, the abovementioned assumptions affect the prediction of the interaction between the particles and the gaseous phase thus limiting the accuracy of the calculations. 

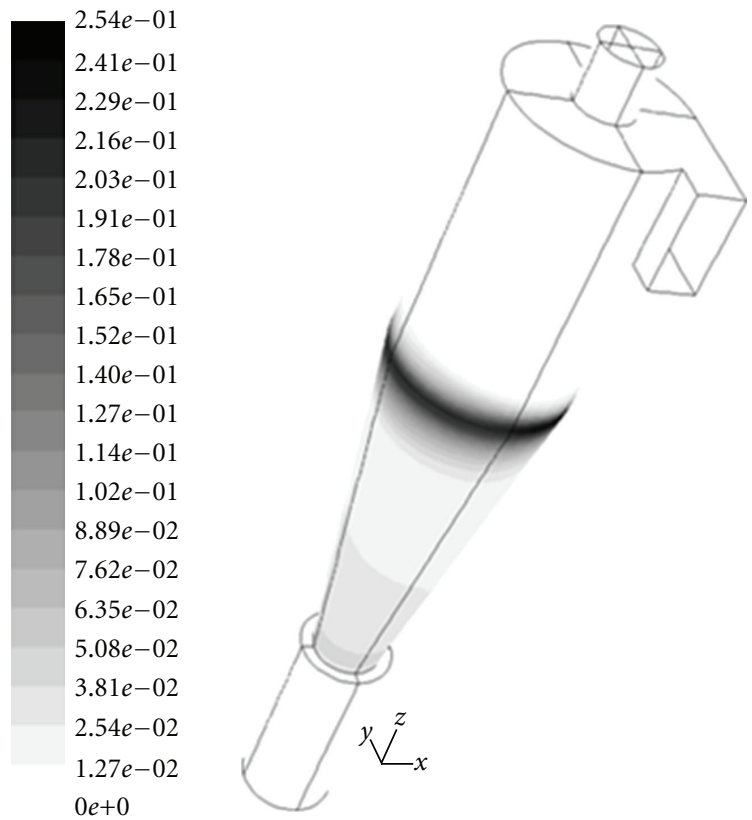

Figure 9: Particles volume fraction near the cyclone wall $\left(1 \mathrm{~kg} / \mathrm{m}^{3}\right)$.

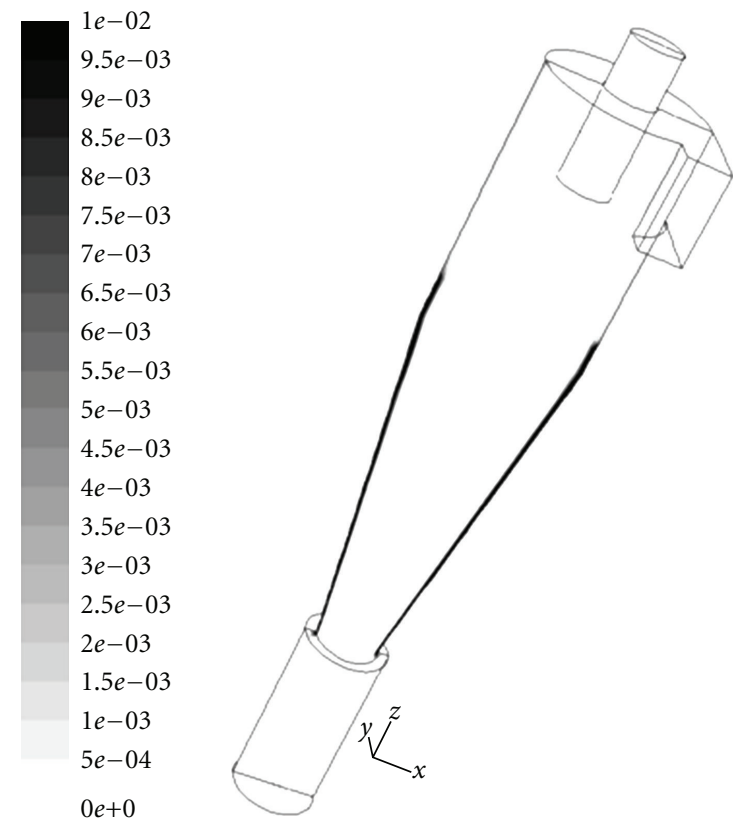

Figure 10: Particles volume fraction at $y=0\left(1 \mathrm{~kg} / \mathrm{m}^{3}\right)$.

From recent studies using the LES turbulence model $[16,36]$, it appears that, although LES gave better results compared with other turbulence models, the improvement sought necessitates more elaborated multiphase models with less simplifying assumptions. One important modeling detail not mentioned in previous CFD works is the accumulation of solid particles in the bottom of the cyclone forming a layer of solid particles which could gradually, as seen in the present work (Figure 12), occupy more than $20 \%$ of the cyclone internal volume during the iterative solution process

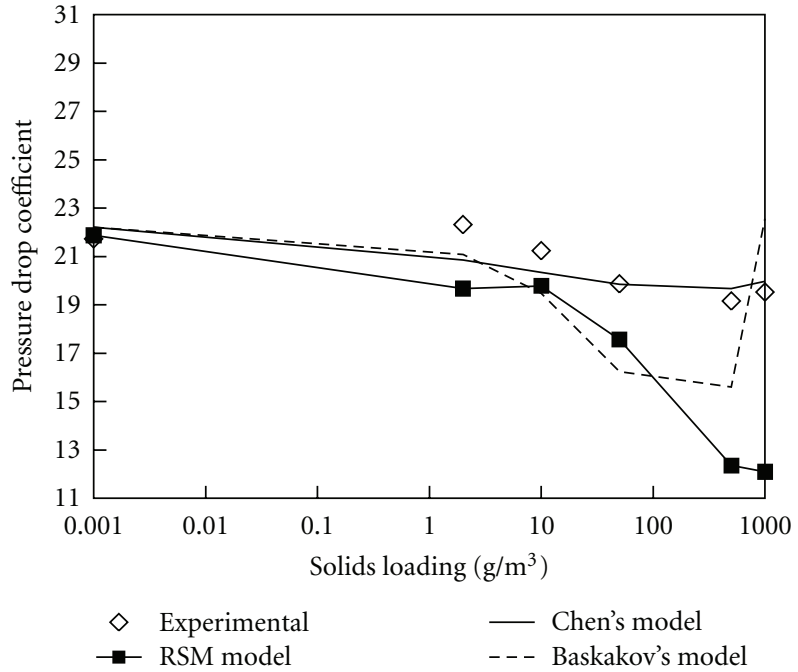

FIGURE 11: Effect of the particles loading on the pressure drop (geometry PV2 in Table 1).

in steady flow or after a number of time steps causing the available working space inside the cyclone to shrink. This was overcome, in the present study, by applying the patch option, available in Fluent, to empty the dust bin periodically during the iterative solution process. The patch option sets the particles volume fraction to zero in the dust bin space. Using the same mesh used for the pure gas case and for the same inlet velocities, another problem encountered was the large values of the dimensionless distance $y^{*}$ (wall function variable) which reached 50000 for concentrations up to $1 \mathrm{~kg} / \mathrm{m}^{3}$. Indeed, the use of standard wall function for the multiphase flow was found to be inappropriate especially at high particle concentrations because the contribution of the solid phase density increased the mixture density and, hence, the $y^{*}$ parameter.

\section{Conclusions}

The Reynolds stress and the granular mixture models have been used to simulate the flow inside a series of standard solid gas cyclones. Pure gas, at different temperatures, and particle-laden flows have been investigated. The mixture model could, reasonably, predict the pressure drop at low concentration of particles in the feed (less than $0.1 \mathrm{~kg} / \mathrm{m}^{3}$ ).

The pressure drop depends strongly on the tangential velocity field. Indeed, the predicted profiles of the tangential velocity component showed that CFD results agreed very well with the experimental results in the absence of particles which explains the reasonable values obtained for the pressure drop in the case of pure gas. On the other hand, increasing the particle loading caused a decrease of the pressure drop corresponding to a damping of the tangential velocity component.

The behavior of the particles inside the cyclone was described using the volume fraction of the solid phase in different regions of the device. High concentrations were observed near the cyclone wall especially in the corners were 

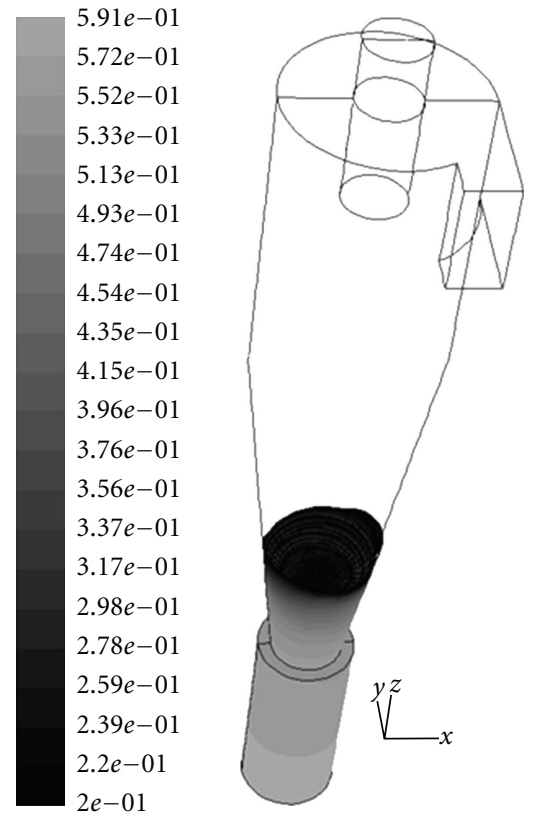

FIGURE 12: Contours of talc volume fraction (accumulation in the cyclone bottom).

a boundary layer exists and where the flow is damped due to high viscous effect.

The use of the entropy generation concept was found to be a useful tool to detect regions of high frictional effects. The interface separating the inner and the outer swirling streams is one of the regions containing important frictional effects.

The granular mixture model presented some limitations for the particle-laden flow which are probably related to the phase coupling. It is noteworthy to mention that the phase coupling especially at high loadings is still a research subject under development. It is possible that the use of the full Eulerian-Eulerian model in conjunction with a robust turbulence model such as large eddy simulation could provide more accurate results and more stable solutions.

\section{Nomenclature}

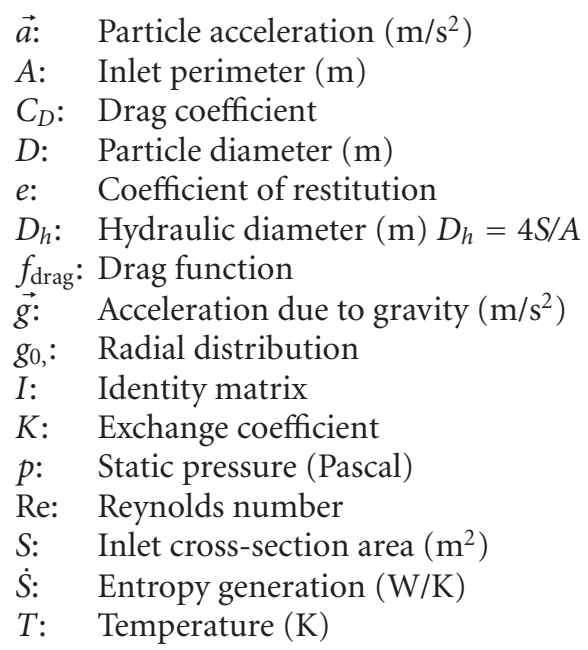

$V_{i}$ : Average inlet velocity $(\mathrm{m} / \mathrm{s})$

$\vec{v}:$ Mass-averaged velocity $(\mathrm{m} / \mathrm{s})$

$\vec{v}_{s}:$ Slip velocity $(\mathrm{m} / \mathrm{s})$

$x$ : Volume fraction

$x_{i}$ : Coordinate $(\mathrm{m})$

$y$ : Mass fraction

Greek Symbols

$\theta: \quad$ Granular temperature $\left(\mathrm{m}^{2} / \mathrm{s}^{2}\right)$

$\mu$ : Laminar viscosity of the mixture $(\mathrm{kg} / \mathrm{ms})$

$\xi: \quad$ Euler number

$\rho$ : Mixture density $\left(\mathrm{kg} / \mathrm{m}^{3}\right)$

$\mu_{t}$ : Turbulent viscosity of the mixture $(\mathrm{kg} / \mathrm{ms})$

$\sigma_{D}$ : Prandtl dispersion coefficient

$\tau$ : Particle relaxation time (s)

$\Phi$ : Kinetic energy exchange

\section{Subscripts}

a: Air (gaseous phase)

D: Dispersion

dr: Drift

$i$ : Direction $i$

in: Inlet

$s$ : Solid particles

sl: Slip.

\section{Acknowledgment}

Authors acknowledge financial support to N. Kharoua from the Petroleum Institute.

\section{References}

[1] Y. Zhu and K. W. Lee, "Experimental study on small cyclones operating at high flowrates," Journal of Aerosol Science, vol. 30, no. 10, pp. 1303-1315, 1999.

[2] T. Chmielniak and A. Bryczkowski, "Method of calculation of new cyclone-type separator with swirling baffle and bottom take off of clean gas - part I: theoretical approach," Chemical Engineering and Processing, vol. 39, no. 5, pp. 441-448, 2000.

[3] P. A. Funk, S. E. Hughs, and G. A. Holt, "Entrance velocity optimization for modified dust cyclones," Journal of Cotton Science, vol. 4, no. 3, pp. 178-182, 2000.

[4] J. Dirgo and D. Leith, "Design of cyclone separators," in Encyclopedia of Fluid Mechanics, 4: Gas-Solid Flows, N. P. Cheremisinoff, Ed., pp. 1281-1306, Gulf Publishing, Houston, Tex, USA, 1986.

[5] C. Cortés and A. Gil, "Modeling the gas and particle flow inside cyclone separators," Progress in Energy and Combustion Science, vol. 33, no. 5, pp. 409-452, 2007.

[6] A. C. Hoffmann and L. E. Stein, Gas Cyclones and Swirl Tubes, Springer, Berlin, Germany, 2nd edition, 2008.

[7] J. Chen and M. Shi, "A universal model to calculate cyclone pressure drop," Powder Technology, vol. 171, no. 3, pp. 184191, 2007.

[8] F. Boysan, W. H. Ayer, and J. Swithenbank, "Fundamental mathematical modeling approach to cyclone design," Transactions of the Institution of Chemical Engineering, vol. 60, pp. 222-230, 1982. 
[9] W. D. Griffiths and F. Boysan, "Computational fluid dynamics (CFD) and empirical modelling of the performance of a number of cyclone samplers," Journal of Aerosol Science, vol. 27, no. 2, pp. 281-304, 1996.

[10] H. F. Meier and M. Mori, "Gas-solid flow in cyclones: the Eulerian-Eulerian approach," Computers and Chemical Engineering, vol. 22, no. 1, pp. S641-S644, 1998.

[11] J. Gimbun, T. G. Chuah, T. S. Y. Choong, and A. Fakhru'lRazi, "Prediction of the effects of cone tip diameter on the cyclone performance," Journal of Aerosol Science, vol. 36, no. 8, pp. 1056-1065, 2005.

[12] J. Gimbun, T. G. Chuah, A. Fakhru'l-Razi, and T. S. Y. Choong, "The influence of temperature and inlet velocity on cyclone pressure drop: a CFD study," Chemical Engineering and Processing, vol. 44, no. 1, pp. 7-12, 2005.

[13] J. Gimbun, "CFD simulation of aerocyclone hydrodynamics and performance at extreme temperature," Engineering Applications of Computational Fluid Mechanics, vol. 2, pp. 22-29, 2008.

[14] B. Wang, D. L. Xu, K. W. Chu, and A. B. Yu, "Numerical study of gas-solid flow in a cyclone separator," Applied Mathematical Modelling, vol. 30, no. 11, pp. 1326-1342, 2006.

[15] F. Qian, J. Zhang, and M. Zhang, "Effects of the prolonged vertical tube on the separation performance of a cyclone," Journal of Hazardous Materials, vol. 136, no. 3, pp. 822-829, 2006.

[16] H. Shalaby, K. Wozniak, and G. Wozniak, "Numerical calculation of particle-laden cyclone separator flow using LES," Engineering Applications of Computational Fluid Mechanics, vol. 2, pp. 382-392, 2008.

[17] V. A. Ivanov, F. J. Sarasola, and S. A. Vasquez, "Multiphase mixture model applied to cyclone separators and bubble columns," American Society of Mechanical Engineers, PressureVessels and Piping Division (Publication) PVP, vol. 397, pp. 317-324, 1999.

[18] M. Narasimha, M. S. Brennan, and P. N. Holtham, "A review of CFD modelling for performance predictions of hydrocyclone," Engineering Applications of Computational Fluid Mechanics, vol. 1, pp. 109-125, 2007.

[19] F. Qian, Z. Huang, G. Chen, and M. Zhang, "Numerical study of the separation characteristics in a cyclone of different inlet particle concentrations," Computers and Chemical Engineering, vol. 31, no. 9, pp. 1111-1122, 2007.

[20] J. J. Derksen, H. E. A. Van Den Akker, and S. Sundaresan, "Two-way coupled large-eddy simulations of the gas-solid flow in cyclone separators," AIChE Journal, vol. 54, no. 4, pp. 872-885, 2008.

[21] M. Manninen, V. Taivassalo, and S. Kallio, On the Mixture Model for Multiphase Flow, Valtion Teknillinen Tutkimuskeskus, Espoo, Finland, 1996.

[22] ANSYS, Inc., "Fluent User Guide and Fluent Theory Guide, version 12.0," 2009.

[23] M. Syamlal and T. J. O’Brien, "Computer simulation of bubbles in a fluidized bed," AICHE Symposium Series, vol. 85, no. 270, pp. 22-31, 1989.

[24] J. Ding and D. Gidaspow, "Bubbling fluidization model using kinetic theory of granular flow," AIChE Journal, vol. 36, no. 4, pp. 523-538, 1990.

[25] C. K. K. Lun, S. B. Savage, D. J. Jeffrey, and N. Chepurniy, "Kinetic theories for granular flow: inelastic particles in Couette flow and slightly inelastic particles in a general flowfield," Journal of Fluid Mechanics, vol. 140, pp. 223-256, 1984.
[26] C. Crowe, M. Sommerfeld, and Y. Tsuji, Multiphase Flows with Droplets and Particles, CRC Press, New York, NY, USA, 1998.

[27] D. Gidaspow, R. Bezburuah, and J. Ding, "Hydrodynamics of circulating fluidized beds, kinetic theory approach," in Proceedings of the 7th Engineering Foundation Conference on Fluidization, pp. 75-82, 1992.

[28] J. M. Dalla Valle, Micromeritics, Pitman, London, UK, 1948.

[29] J. R. Richardson and W. N. Zaki, "Sedimentation and fluidization: part I," Transactions of the Institution of Chemical Engineers, vol. 32, pp. 35-53, 1954.

[30] B. E. Launder, G. J. Reece, and W. Rodi, "Progress in the development of a Reynolds-stress turbulence closure," Journal of Fluid Mechanics, vol. 68, no. 3, pp. 537-566, 1975.

[31] B. E. Launder and D. B. Spalding, "The numerical computation of turbulent flows," Computer Methods in Applied Mechanics and Engineering, vol. 3, no. 2, pp. 269-289, 1974.

[32] F. Boysan, J. Swithenbank, and W. H. Ayers, "Mathematical modelling of gas-particle flows in cyclone separators," in Encyclopedia of Fluid Mechanics, Vol. 4, N. P. Cheremisinoff, Ed., pp. 1307-1329, Gulf Publishing, Houston, Tex, USA, 1986.

[33] T. H. Ko, "Numerical analysis of entropy generation and optimal Reynolds number for developing laminar forced convection in double-sine ducts with various aspect ratios," International Journal of Heat and Mass Transfer, vol. 49, no. 3-4, pp. 718-726, 2006.

[34] A. P. Baskakov, V. N. Dolgov, and Y. M. Goldobin, "Aerodynamics and heat transfer in cyclones with particle-laden gas flow," Experimental Thermal and Fluid Science, vol. 3, no. 6, pp. 597-602, 1990.

[35] L. Xiaodong, Y. Jianhua, C. Yuchun, N. Mingjiang, and C. Kefa, "Numerical simulation of the effects of turbulence intensity and boundary layer on separation efficiency in a cyclone separator," Chemical Engineering Journal, vol. 95, no. 1, pp. 235-240, 2003.

[36] J. J. Derksen, S. Sundaresan, and H. E. A. van den Akker, "Simulation of mass-loading effects in gas-solid cyclone separators," Powder Technology, vol. 163, no. 1-2, pp. 59-68, 2006. 

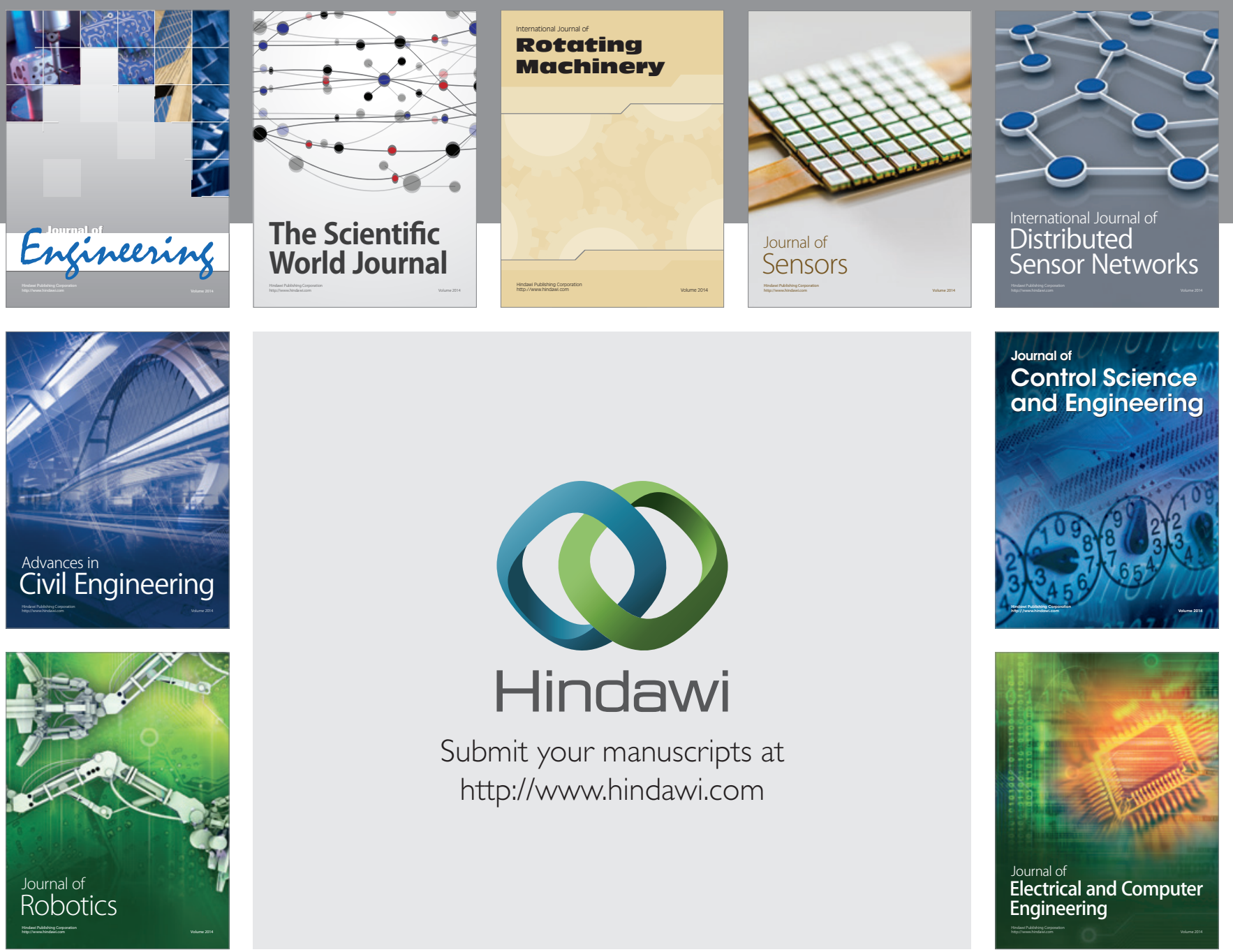

Submit your manuscripts at

http://www.hindawi.com
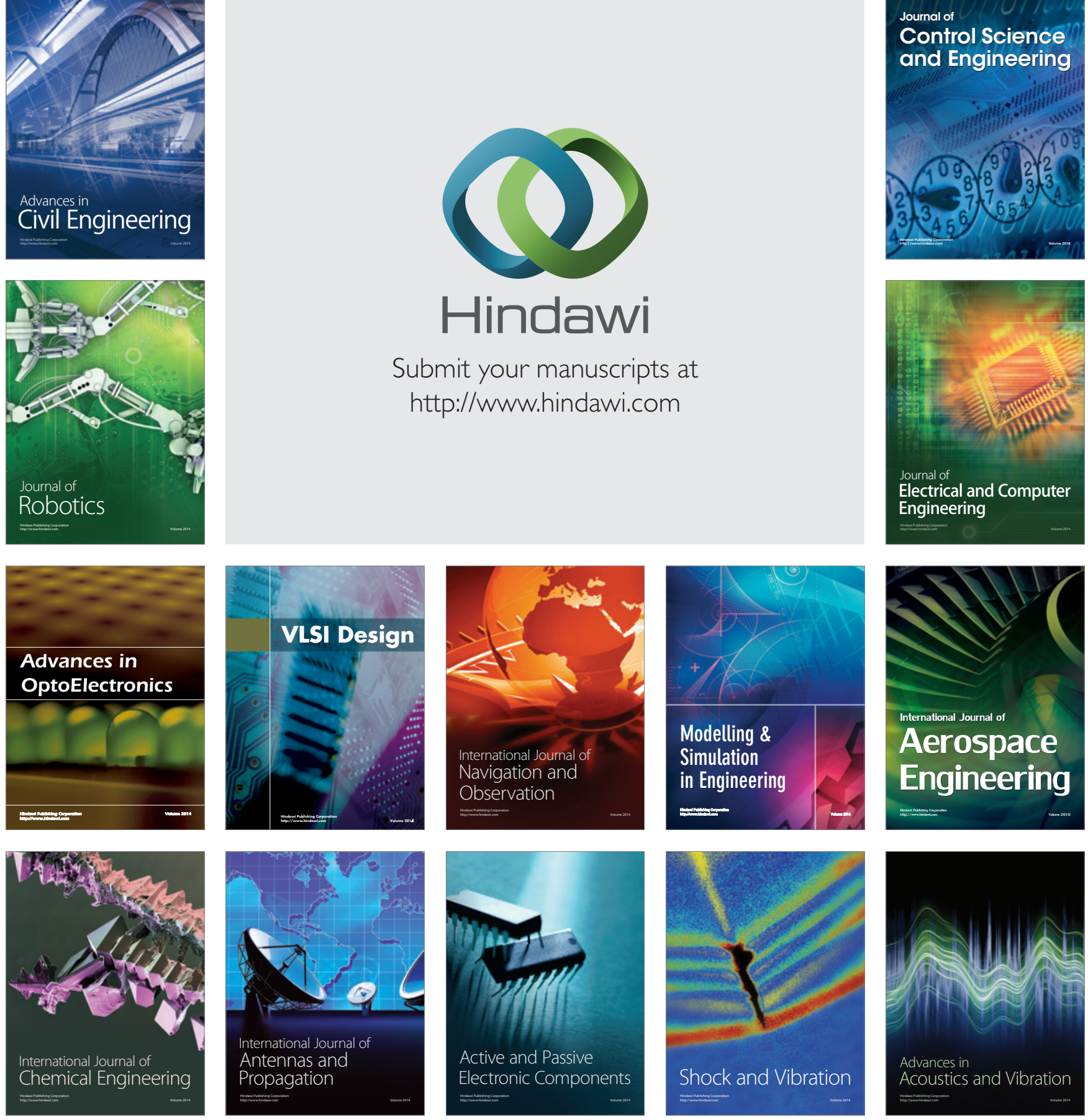